\title{
Extraction of a translocated piece of an implantable cardioverter-defibrillator lead from the hepatic vein
}

\author{
Artur Oręziak ${ }^{1}$, Anna Wojno ${ }^{2}$, Adam Parulski ${ }^{3}$, Maciej Sterliński ${ }^{1}$ \\ ${ }^{1}$ Department of Arrhythmia, Institute of Cardiology, Warsaw, Poland \\ ${ }^{2}$ Department of Anesthesiology, Institute of Cardiology, Warsaw, Poland \\ ${ }^{3}$ Department of Cardiac Surgery and Transplantology, Institute of Cardiology, Warsaw, Poland
}

Transvascular lead extraction (TLE) is associated with technical limitations and complications, including fragmentation of the lead body and its uncontrolled translocation.

This article reports the case of a 50 -year-old female with an atrial valve replaced 32 years ago, paroxysmal atrial fibrillation, congestive heart failure with a $40 \%$ ejection fraction, and Epic VR (St. Jude Medical, USA) implantable cardioverterdefibrillator (ICD) with a Linox S 65 single-coil active defibrillation lead (Biotronik, Germany) implanted in 2009. She was referred for TLE because of damage to the defibrillation lead.

During TLE, the defibrillation lead insulation was pulled out below the inserted Liberator locking stylet (Cook Medical, USA), stretching the coil-wire and leaving the distal part of the lead (Fig. 1A). Attempts to remove the remains of the lead through femoral access using a Needle's Eye Snare (Cook Medical, USA) were unsuccessful the coil-wire was torn and a loose fragment trans- located to the hepatic vein (Fig. 1B). The broken lead fragment was successfully removed using a bioptome (Jawz ${ }^{\mathrm{TM}}$ Endomyocardial Biopsy Forceps, Argon Medical Devices, USA) which was inserted through the transseptal Agilis ${ }^{\mathrm{TM}} \mathrm{NxT}$ steerable introducer (SJM, USA) placed in the hepatic vein (Fig. 1C, Suppl. Video 1).

Fragmented parts of extracted leads may be a source of venous embolism. The real clinical significance of a small metallic wire left in the hepatic vein is still unknown with respect to potential infectious and mechanical consequences, but it was shown that the decision to remove the wire from the hepatic vein was effective and safe (Fig. 1D).

Conflict of interest: Artur Oręziak - fees from St. Jude Medical, Medtronic and Biotronik; Maciej Sterliński - fees from Hammermed (Cook), Medtronic, St. Jude Medical and Biotronik; Anna Wojno and Adam Parulski — none declared.

Address for correspondence: Artur Oręziak, MD, PhD, Department of Arrhythmia, Institute of Cardiology, ul. Alpejska 42, 04-628 Warszawa, Poland, tel/fax: +48 603374235, +48 22 3434417/+48 22 3434520, e-mail: aoreziak@ikard.pl 


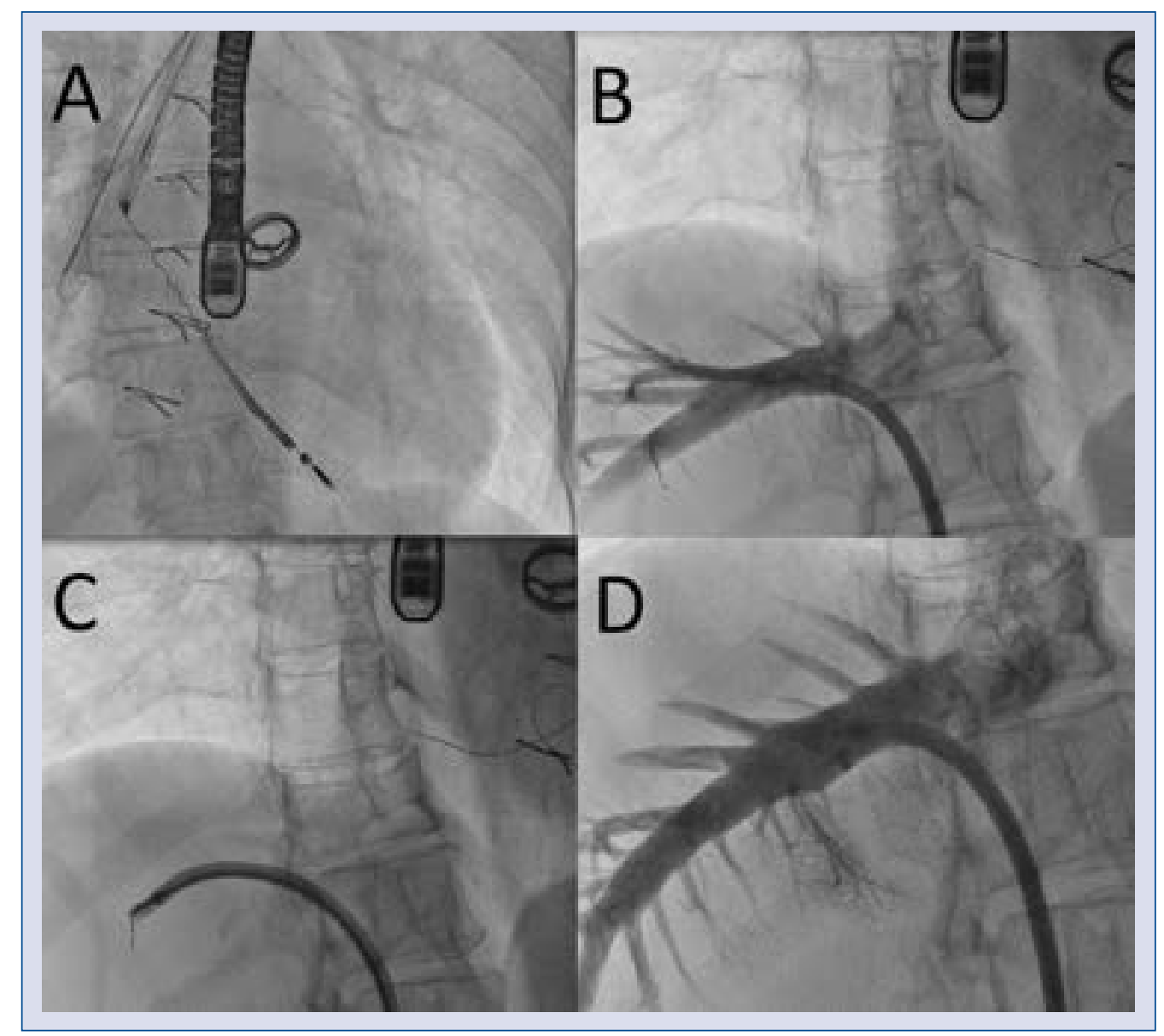

Figure 1. A. The stretched coil-wire during transvascular lead extraction of the defibrillation lead (right anterior oblique view [RAO] $25^{\circ}$ ); B. Venography of the hepatic veins with the translocated fragment of the metal coil-wire (RAO $\left.25^{\circ}\right)$; C. Removal of the fragment of the metal coil-wire from the hepatic vein using the bioptome in the steerable introducer (RAO $25^{\circ}$ ); D. Venogram of the hepatic veins after removal of the part of metal coil-wire (RAO $25^{\circ}$ ). 